\title{
The Rotting Meat Error: From Galileo to Aristotle in Data Mining?
}

\author{
Giovanni Comandé*
}

\section{The Rotting Meat Error}

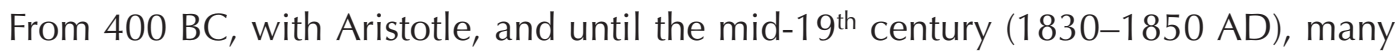
scientists have claimed that some organisms can be generated spontaneously from non-living matter, citing larvae as an example and the flies that are generated on decomposing meat.

Aristotle had observed the correlation between the rotting of meat, the appearing of larvae on it and the developing of flies. Upon his repeated observations, he found a correlation that developed in a theory (the theory of spontaneous generation). This theory proved wrong (the 'rotting meat error' in our story) because it grew out of correlations 'statistically' (very) significant but those correlations were wrong ones. Yet, for a long time the theory was constantly applied as 'scientific' understanding since Aristotelian logic applied well to it.

Only in 1600, Francesco Redi contradicted the theory with his experiments beginning to break the uninterrupted chain of observations correlating rotting meat and larvae generation, a chain finally severed by Luis Pasteur in the $19^{\text {th }}$ century.

Between Aristotle and Redi the scientific method developed by Galileo Galilei was born, putting between observed correlations and conclusions the need to find a causal explanation for them and to prove it by experiments.

Aristotle moved from observations, correlating them in syllogisms. According to him, syllogism is a discourse in which, once established some variables, something different from what is established follows on from necessity by virtue of what is established. Thus, there is a demonstration when the syllogism proceeds from true and raw assertions, or from assertions that have assumed the principle of knowledge related to them by virtue of certain true and prime assertions. Dialectic is instead the syllogism that argues from remarkable opinions.

It is easy to notice the resemblance between Aristotle's approach and modern data mining. 
Of course, we are aware that data mining is the computational process of discovering patterns in large data sets involving methods at the intersection of artificial intelligence, machine learning, statistics, and database systems. Also, we know that the overall goal of the data mining process is to extract information from a data set and transform it into an understandable structure for further use; what we normally call a pattern. However, the true goal of data mining is not the extraction (mining) of data itself but the extraction of patterns and knowledge from large amounts of data ${ }^{1}$. Data are like the nature observed by Aristotle. Patterns are consolidated observations (to echo Aristotle) that become knowledge when they are put in use assuming they reflect a truth.

These are important qualifications that must be clarified because hidden in the process is a deep epistemological revolution in data mining with enormous implications.

As in Aristotle, pattern emanates from observations (in data mining powered by Al, informatics and statistics). Algorithms weight the relevance and reliability of those patterns mathematically; often machine learning generated algorithms are at work for these tasks. Once the correlations assume the 'principle of knowledge', patterns are used in the decision-making processes as they were in syllogisms. Note, however, that the decision to assign to patterns the 'principle of knowledge' is possibly taken by an entity different from the one developing the pattern used itself.

As it has been nicely summarised:

In data mining, a pattern is a statement that describes relationships in a (sub) set of data such that the statement is simpler than the enumeration of all the facts in the (sub) set of data. When a pattern in data is interesting and certain enough [emphasis added] for a use, according to the user's criteria [emphasis added], it is referred to as knowledge. Patterns are interesting when they are novel (which depends on the user's knowledge), useful (which depends on the user's goal), and nontrivial to compute... A pattern is not likely to be true across all the data. This makes it necessary to express the certainty of the pattern. Certainty may involve several factors, such as the integrity of the data and the size of the sample. ${ }^{2}$

Thus, according to Aristotle's logic, maybe not all patterns are wrong, but certainly some are still useful although wrong. For instance, wrong patterns and wrong theories (eg Ptolemy on flat earth) were able to explain consistently the world around us for some time and enabled humanity to evolve and travel in non-flat earth, for instance.

Similarly, users of data mining outputs could be willing to use these results although aware that the output might not be correct. Actually, without even willing to know whether they are correct or not. In the era of big data, it has been suggested,

1 Tal Z Zarsky, "Mine your own Business!": Making the Case for the Implications of the Data Mining of Personal Information in the Forum of Public Opinion' (2003) 5 Yale Journal of Law and Technology 1.

2 Bart Custers, 'Data dilemmas in the information society: introduction and overview' in Bart Custers et al (eds), Discrimination and Privacy in the Information Society: Data Mining and Profiling in Large Databases (Springer 2013). 
Google's founding philosophy is that we don't know why this page is better than that one: If the statistics of incoming links say it is, that's good enough. No semantic or causal analysis is required. That's why Google can translate languages without actually 'knowing' them (given equal corpus data, Google can translate Klingon into Farsi as easily as it can translate French into German). And why it can match ads to content without any knowledge or assumptions about the ads or the content. ${ }^{3}$

Consequently, it is suggested that in the big data era the entire scientific method is called into question since the potential richness to harness in big data is (supposed to be) bigger than the fruits of searching for causality. The rotting meat error, however, offers the occasion to question the generality of this assertion.

The last quote illustrates clearly the collapse of the distinction (to date clearly made by scientists) between correlation and causation: the gist of the scientific approach for which after observation of data we-subsequently-first hypothesise, then create a model, testing afterwards this model before applying it to given contexts for which it has been tested!

Consequences of such a collapse switching from Galileo to Aristotle can be far-reaching and require some warning.

\section{Going Back to the Future? Some Reasons to Be Cautious}

The rotting meat error is potentially more disruptive in the era of big data than it was for centuries under the Aristotelian approach to correlations.

The way correlations are generated today is strictly dependent on various factors among which here we briefly consider the quality and quantity of data, their closeness ${ }^{4}$ to their actual significance in relation to the data subject, on the one hand, and on the other, the quality of the algorithm(s) used in their generation and analysis.

Let us concentrate for a moment on the algorithm(s). Here we mean the different potential tools used to mine and analyse data or to put the results into use as knowledge.

Data are 'generated' in a context ${ }^{5}$ but they are not given meaning only in that context. For instance, data on my physical activities (eg average number of steps walked daily) have per se a meaning depicting me with accuracy as an active/inactive person. The very same data, illustrating the average number of steps I walk every day during a cer-

3 Chris Anderson, 'The End of Theory: The Data Deluge Makes the Scientific Method' Wired (2008) < https://www.wired.com/2008/06/pb -theory/> accessed 15 August 2018.

4 Giovanni Comandé and Gianclaudio Malgieri, 'Sensitive-by-distance: Quasi-health Data in the Algorithmic Era' (2017) 3 Information, Communication and Tehcnology Law 1.

5 See for the risks of de-contextualisation Helen Nissembaum, Privacy in Context: Technology, Policy, and the Integrity of Social Life (Stanford University Press 2010). 
tain period can be correlated to other data, either related to me like the classes I teach or attend, or totally unrelated, such as the activities or events registered for people that share with me this given characteristic (eg average number of steps daily).

Once personal data are used to correlate to a model not built on my own data their closeness to their actual significance in relation to the data subject is diminishing. Yet, those correlations are extensively used in every activity opening the way to the rotting meat error, that is, assuming a correlation as valid without testing it.

Assume that a certain number of people attending my classes declare they are deeply bored by them. Assume also that a number of my students later discover to have contracted a specific illness. Assume that also other people who declared to be deeply bored on other occasions have contracted the same illness although did not attend my classes. Finally assume that other people who did not get bored at all (regardless of the attendance of my courses) contracted the same illness. Now a data mining process in a vast ocean of data containing as well these variables finds easily these correlations:

1) a certain number of individuals $(x, y, z, w, \ldots)$ who got deeply bored have contracted illness X (eg diabetes);

2) all bored individuals $(x, z, w, \ldots)$ who attended Comandè's classes have contracted diabetes;

3) some individuals ( $x, y, z, w, a, b, f, s, d, g, \ldots)$ who spent a '?' amount of time in location ' $\mathrm{L}$ ' (incidentally, Comandè's classroom is in this geographical area) have contracted diabetes;

4) a number of individuals who did not attend Comandè's class have contracted diabetes as well.

Now, depending on the way the correlations are weighted, an algorithm can easily infer from the above 'true and raw assertions' that Comandè's classes are both boring and correlated to illness X. Even admitting that my classes are boring, it remains visible that they cannot cause diabetes, what has been found is a simple correlation, a proxy. However, these correlations are released or available in the ocean of data. It remains on their final user to determine the actual usage they are put to; to find them 'interesting and certain enough for a use, according to the user's criteria', that is to make use of this information for a certain action.

Hence, assume that an algorithm must suggest to you whom to hire for a job among people with the same qualification and experience. Yet, from the CV of one of them emerges that she attended my classes. It is possible that the algorithm gives a (significant) weight to variable 2 above; a weight that is really small but that tips the balance against my former student (for the risk of developing diabetes incurring extra costs related to, eg, healthcare expenditures, or more absences from work sometime in the fu- 
ture). This generates an inconvenient rotting meat error significantly affecting my student.

Lack of transparency on the methods employed by users in deciding an information is certain enough for a use, and the weight it has in the decision-making process accord-

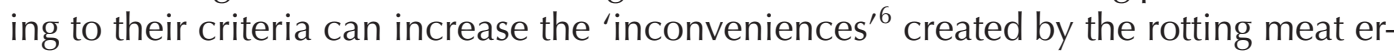
ror. These inconveniences can produce 'legal effects' concerning the data subject 'or similarly significantly affects him or her', to use the language of Article 22 of the General Data Protection Regulation (GDPR) on solely automated decision-making that cannot easily be cured.

In addition, the use of these methods has certainly an impact ${ }^{7}$ on our decision-making context that is yet to be fully explored.

Let us now concentrate more on data closeness and their original significance because it influences the protective scope of Articles 9 and 22 of the GDPR.

\section{The Interplay between Data Mining and Inflected Data}

Data mining allows the inflection of data in various directions.

We will refer here to inflected data every time data of a certain significance (eg geolocalisation, type or quantity of food intake...) lend themselves to generate other data of different significance (inflect the original data in a different direction). It may be the example of raw data such as 'steps walked', 'sugar intake', 'location' that jointly with other data generate new data with a different significance (eg health related) literally inflecting the original significance of the raw data in a different direction.

The enormous amount of data created every second by the 'technological extensions' of individuals, by the expanding number of sensor data and Internet of Things entities directly or indirectly reconnected to individuals make possible this phenomenon. Indeed, an enormous and relentlessly growing market of collecting, storing, processing and reselling data from various information sources ${ }^{8}$ has been enabled by the potential of combining different datasets through mining techniques.

Certainly, it is the goal of data mining to extract from the analysed datasets hidden information and productive correlations ${ }^{9}$ to find new patterns and relations in that

6 Oscar H Gandy Jr, 'Engaging Rational Discrimination: Exploring Reasons for Placing Regulatory Constraints on Decision Support Systems' (2010) 12 Journal of Ethics of Information Technology 29, 35; Solon Barocas and Andrew D Selbst, 'Big Data's Disparate Impact' (2016) 104 California Law Review 671.

7 Daniel Solove, "I've Got Nothing to Hide" and Other Misunderstandings of Privacy' (2007) 44 San Diego Law Review 745; Danielle Keats Citron and Frank Pasquale, 'The scored society: due process for automated predictions' (2014) 89 Washington Law Review 1.

8 Julie E Cohen, Configuring the Networked Self: Law, Code, and the Play of Everyday Practice (Yale University Press 2012).

9 ibid $40-43$. 
data. The databases used are large both in size-they may contain millions of records - and in the variety of types of data. In addition, data mining usually does not begin with a hypothesis, but instead uses a variety of tools to generate hypotheses and test them against the available data, data whose closeness to the original collection is continuously diluted at every addition, integration, exchange of the dataset they are included in. These processes can easily become self-referential finding validations in the same dataset that generated them, but we cannot deal here with this issue.

Data mining processes reveal patterns or create group profiles through algorithms that cluster data into groups with similar properties; classify data by mapping them onto pre-defined classes, or describing correlations through regression analysis ${ }^{10}$. These pigeonholing processes proved often very useful and valuable in the many domain of application 'growing respect for correlations rather than a continuing quest for elusive causality ${ }^{11}$ because of their capability to 'uncover hidden patterns and subtle relationships in data, and to infer rules that allow for the prediction of future results' ${ }^{12}$

Yet if we consider that patterns are hypotheses automatically generated by algorithms based on the premises given by a certain set of data ${ }^{13}$ some reasons of concern can emerge. These hypotheses can derive from direct matches, especially when data used are close to their original significance or from probabilistic links or predictive guesses. ${ }^{14}$ Following our example of diabetes, beyond direct match to an illness (eg registered in a medical record), there can be a probabilistic link to it (eg family history) or a predictive guess (eg based on dietary habits, lifestyle, attendance of Comandé's classes). Note that these predictive guesses are also inferred data that is data that cannot be deduced logically from the available provided and observed data. A human being could not generate them without the support of intelligent systems relying on probability-based analytic tools. ${ }^{15}$ This is a strength of data science that must be appreciated with the cautions suggested by the rotting meat error.

As we illustrated elsewhere ${ }^{16}$ for health data this is a continuous iterative cycle for the identification of new patterns with increasingly weaker connections-reduced

10 Giovanni Comandé, 'Regulating Algorithms' Regulation? First Ethico-Legal Principles, Problems and Opportunities of Algorithms' in Tania Cerquitelli, Daniele Quercia and Frank Pasquale (eds), Transparent Data Mining for Small and Big Data (Springer 2017).

11 Viktor Mayer-Schönberger and Kenneth Cukier, Big Data: A Revolution That Will Transform How We Live, Work, and Think (John Murray Publishers 2013) [23].

12 Zarsky (n 1) 6-7.

13 These hypotheses are generated by recognition algorithms that search for variables typically matching with each other on account of association rules and link analyses. See Mireille Hildebrandt, 'Defining Profiling: A New Type of Knowledge?' in Mireille Hildebrandt and Serge Gutwirth (eds), Profiling the European Citizen- Cross-disciplinary Perspectives (Springer 2008) [17-30].

14 See Lokke Moerel and Corien Prins, 'Privacy for the Homo Digitalis: Proposal for a New Regulatory Framework for Data Protection in the Light of Big Data and the Internet of Things' (SSRN, 25 May 2016) < https://ssrn.com/abstract=2784123> or <http://dx.doi.org/10.2139/ssrn.2784123 $>$ accessed 15 August 2018.

15 See Organisation for Economic Co-operation and Development, Report on Data-Driven Innovation for Growth and Well-being, (OECD 2015) [65] <http://www.oecd.org/sti/inno/data-driven-innovation-interim-synthesis.pdf>accessed 15 August 2018.

16 Giovanni Comandé and Giulia Schneider, 'Regulatory Challenges of Data Mining Practices: The Case of the Never-ending Lifecycles of 'Health Data' (2018) 25 European Journal of Health Law 284. 
closeness - to the original significance of the data ${ }^{17}$ increasing the risk of rotting meat errors.

\section{Warning from the Example of Health-Inflected Data}

We have argued elsewhere ${ }^{18}$ that in the current technical environment nearly every type of data circulating in the market is actually or potentially health-inflected. The massive availability of various health-related data has in turn ended up broadening the health-grounded basis of decisions taken far outside the healthcare sphere, feeding in this way spillover uses of health data that drive classifications and, with that, rules of inclusion and exclusion from society. At a more general level of analysis, the case of health data shows that data mining techniques challenge core data-protection notions, such as the distinction between sensitive and non-sensitive personal data, requiring a shift in terms of systemic perspectives that the GDPR only partly addresses.

Here we claim that the weaker the connection with data of the predictive guess the more problematic it is and, paradoxically, the less it is able to trigger the higher level of protection needed. ${ }^{19}$ A clear example is the use of health-inflected data instead of plain health data, but conclusions can be generalised ${ }^{20}$.

Article 9 GDPR prohibits, with broad exceptions, the processing of 'special categories of data' such as 'data concerning health or data concerning a natural person's sex life or sexual orientation'. We have begun to challenge the strengths and soundness of the current notion of health data ${ }^{21}$ but here we want to recall them as a clear example of the paradigm shift data mining techniques are producing in our way of dealing with truth and causality and how this impacts on the GDPR application.

Furthermore, Article 22 prohibits 'a decision based solely on automated processing, including profiling' 'based on special categories of personal data' unless -1 -with 'explicit consent to the processing of those personal data for one or more specified purposes', or 'for reasons of substantial public interest' - 2 - 'and suitable measures to safeguard the data subject's rights and freedoms and legitimate interests are in place'.

However, health-inflected data-that is, data not necessarily covered by Article 9 - are often used to make decisions regarding data subjects, ${ }^{22}$ blurring the borders between non-sensitive and sensitive personal data and bypassing the protective scope of provisions like Articles 9 and 22. In fact, in the realm of the rotting meat error risks related

7 Comandé and Malgieri (n 4) 22-23.

18 Comandé and Schneider (n 16) 286.

19 Comandé and Malgieri (n 4).

20 Frank Pasquale, The Black Box Society - The Secret Algorithms that Control Money and Information (Harvard University Press 2015).

21 Comandé and Schneider (n 16); Comandé and Malgieri (n 4) 22-23.

22 See Comandé (n 10) 174-176. 
to the processing of a certain dataset are not any more related to the single data subject but rather to the category in which the single data subject is every time systematised based on data generated by other similar subjects. ${ }^{23}$ As illustrated elsewhere, ${ }^{24}$ these classifications are often based on data with deteriorating indirect connection to the data subject. Yet, they are used in many relevant fields - from direct advertisements and marketing ${ }^{25}$ to credit issuing and employment ${ }^{26}$ - leading to differential economic treatments that can extensively lead to rotting meat errors difficult to identify and remedy.

In this continuous process generating the classifying society, ${ }^{27}$ associative and probabilistic analytics transform runaway data of every sort-eg from financial transactions to statistical data referring to crime rates or house prices, GPS information or lifestyleinto silent and unexpected determinants of decisions. The signalling power provided by these analytics and the expounding abandonment of Galileo and acceptance to switch to Aristotle overshadows the rotting meat problem and the consequences of accepting mere correlations without looking for causal explanations. A difficulty we think worthy of further exploration.

23 Lanah Kammourieh et al, 'Group Privacy in the Age of Big Data' in Linnet Taylor, Luciano Floridi and Bart van der Sloot (eds), Group Privacy-New Challenges of Data Technologies (Springer 2016) 37-66.

24 Comandé and Malgieri ( $\mathrm{n}$ 4).

25 Executive Office of the President of the United States, 'Big Data: Seizing Opportunities, Preserving Values' (February 2015) [4-6] <https:// obamawhitehouse.archives.gov/sites/default/files/docs/big_data_privacy_report_may_1_2014.pdf > accessed 15 August 2018; Keats Citron and Pasquale (n 7).

26 Frank Pasquale, The Black Box Society - The Secret Algorithms that Control Money and Information (n 20).

27 Comandé (n 10). 